\title{
PROTECTIVE EFFICACY OF COMMERCIAL NEW CASTLE DISEASE VACCINES AGAINST VIRULENT GENOTYPE VII NEWCASTLE DISEASE VIRUS
}

Hasnaa Ahmed ${ }^{1}$, Mohamed Khodier ${ }^{2}$, Samy Kasem ${ }^{3}$, Abd El-GalilEl-

$$
\text { Gohary }^{4}
$$

${ }^{1}$ Department of Virology, Faculty of Veterinary Medicine,Kafrelsheikh University, 33516, El-Geish Street, Kafrelsheikh, Egypt

${ }^{2}$ Veterinary Serum and Vaccine Research Institute, Abbasia, Cairo, Egypt,

${ }^{3}$ Department of Poultry Diseases, Faculty of Veterinary Medicine, Kafrelsheikh University, 33516, El-Geish Street, Kafrelsheikh, Egypt.

\section{ABSTRACT}

Despite the widespread use of different types of vaccines, Newcastle disease continues to be a major threat in poultry industry that emphasizes the importance of continued research on vaccine efficacy against newly isolated strains. Our study assessed whether a virulent genotype VII NDV field isolate can overcome the immunity of chickens induced by the currently used ND vaccines after different vaccination schemes in Egypt. Two hundred Cobb chicks (one day old) obtained from Alwatania Poultry Company Egypt branch were used, Chicks were divided into four groups ( $n=50$ per group), Group I: Ten chicks used for measuring maternal immunity at day one of age, And 40 chicks kept as a control group, and they were divided into 4 subgroups ten for each, three subgroups (30 chicks) were used as positive control and they were vaccinated with the same vaccines of the experimental groups but without challenge, and the last ten chicks were kept unvaccinated to be used as negative control. Other three groups besides those of group I (control group) were primary 
protective efficacy of commercial new castle disease ...

Hasnaa Ahmed et., al.

immunized with Hitchner-B1 at day 7, then subjected to the following vaccines at day 14 as follow, Group II was immunized via eye dropping with commercial live LaSota vaccine. Group IIII was immunized via eye dropping with commercial live Clone-30 vaccine. Group IV was immunized via eye dropping with commercial live ND 6/10 vaccine. Experimental challenge of the different groups revealed death of all 10 challenged birds of the negative control group, death of 12 out of 50 challenged birds in group-II; 14 out of 50 challenged birds in group-III; 9 out of 50 birds in group-IV. Recent studies and ours revealed that prevalent genotype VII differ from widely used vaccine strains in its genetics, immune response and protective efficacy. So, further studies on antigenic characters of recent NDV isolate and protective efficacy of currently used NDV vaccines against it are needed.

Keywords: Newcastle disease virus, Genotype VII, Egypt

\section{INTRODUCTION}

Newcastle disease (ND) is one of the most contagious of all viral diseases, which has severe negative impacts on poultry industries (Alexander and Senne, 2008). The etiologic agent, Newcastle disease virus (NDV), is an enveloped cytoplasmic virus called avian paramyxovirus serotype1 (APMV-1) which is a member of the genus Avulavirus in the family Paramyxoviridae. The virus is a single-stranded, non-segmented, negative sense RNA genome (Goldhaft, 1980; Dortmans, 2011; Al-Habeeb et al., 2013).

NDV has a single serotype.However, genetic and antigenic diversity are recognized for NDV isolates (Miller et al., 2010). APMV1Two distinct genetic clades, class I and class II, are recognized based on 
the complete or hyper variable nucleotide sequence of the F gene. Class I and class II comprise at least nine (1-9) and ten (I-X) genotypes, respectively. All class I strains except for one strain and most of genotype I and genotype II viruses within class II are of low virulence (Kim et al., 2007a,b). Genotypes I, II, III, IV, and IX were mostly isolated before the 1970s, while the predominant circulating genotypes are V, VI, VII, VIII, and X which emerged since the 1960s and contain only virulent viruses.

Genotype VII is particularly important given that it has been associated with many of the most recent outbreaks in Asia, Africa, and the Middle East (Khan et al., 2010). Despite advances made in diagnosis and vaccination for Newcastle disease, the disease continues to negatively impact on poultry production world-wide (Alexander et al., 2012).

Haemagglutination inhibition (HI) test is most widely used for detecting and identification of the NDV and the most direct factors to estimate the protection induced by ND vaccines (Elham et al., 2014).

Strains Hitcher B1 and LaSota belong to genotype II in class II and were isolated some 60 years ago, whereas the predominant genotypes currently circulating world-wide are genotypes V, VI and VII. Although the current vaccines offer substantial protection from disease, they do not completely prevent the infection or virus shedding, and disease can occur in vaccinated birds. The recent outbreaks of ND in different parts of the world highlight the inadequacy of currently used vaccines. In contrast, recent studies indicated that inactivated vaccines (Miller $\boldsymbol{e t}$ al., 2007; $\mathbf{H u}$ et al., 2011), or a live attenuated vaccine developed from currently 
circulating genotype strains had increased effectiveness (Alexander, 1998).

The present study was designed to evaluate the efficacy of some currently used ND vaccination schemes against NDV genotype VII, as a currently circulating strain.

\section{MATERIALS AND METHODS}

\section{Animals:}

Two hundred Cobb chicks (one day old) obtained from Alwatania Poultry Company Egypt branch were used ,they were divided into four groups ( $\mathrm{n}=50$ per group),Group I: Ten chicks used for measuring maternal immunity at day one of age ,And 40 chicks kept as a control group, and they were divided into 4 subgroups ten for each, three subgroups(30 chicks) were used as positive control and they were vaccinated with the same vaccines of the experimental groups but without challenge as follows they received Hitchner B1 vaccine at 7 dayold via eye dropping followed by poster at day 14 vaccines as follow, first 10 take LaSota vaccine, second 10 take Clone 30 vaccine, third 10 take ND 6/10 vaccine all via eye dropping, and the last ten of them were unvaccinated as negative control .Other three groups besides those of group I (control group) were primary immunized with Hitchner-B1 at day 7 , then subjected to the following vaccines at day 14 as follow, Group II was immunized via eye dropping with commercial live LaSota vaccine. Group IIII was immunized via eye dropping with commercial live Clone-30 vaccine. Group IV was immunized via eye dropping with commercial live ND 6/10 vaccine (Table-1).

One hundred and sixty birds were challenged at 35th day with locally isolated VVNDV (genotype VII) using a dose of $10^{6}$ ELD50 in Kafrelsheikh Vet. Med. J. Vol. 15 No. 1 (2017) 
$0.1 \mathrm{ml} /$ chick inoculated I/M and observed for clinical signs and death for 10 days.

\section{Vaccines and Viruses:}

Asymptomatic ND 6/10 strain which was supplied by Agromed Company and three live lentogenic NDV strains; Hitchner-B1; LaSota; Clone 30 vaccine in freeze dried forms were supplied by Veterinary Serum and Vaccine Research Institute, Abbasia, Cairo. Egypt.

Virulent (genotype VII) locally isolated Velogenic viscerotropic NDV strain with titer of $10^{6} \mathrm{EID}_{50}$ which was kindly obtained from Veterinary Serum Vaccine Research Institute, Abbasia, Cairo used for challenge of experimentally vaccinated chickens.

\section{Evaluation the immune response of chicken by Haemagglutination inhibition (HI) and ELISA.}

To evaluate the immune state of the challenged chicks, sera were collected, at (1-7-14-21-28 and 35 days old). Sera were separated by incubating the clotted blood samples at $4^{\circ} \mathrm{C}$ for $6 \mathrm{~h}$ then centrifugation at $3000 \mathrm{rpm}$ for $10 \mathrm{~min}$. Serum samples were then inactivated at $56{ }^{\circ} \mathrm{C}$ for 30 min.and stored at $-20^{\circ} \mathrm{C}$ until used.Sera samples were examined by Haemagglutination inhibition assay which performed according to standard procedures (Alexander, 1998), using four HA units of antigen. The HI titers were read as the reciprocal of the highest dilution showing complete inhibition. Also sera collected from the experimentally 
vaccinated chickens were also tested for ND antibodies using the ELISA technique as described by (Volleret al., 1976).

\section{RESULTS}

\section{Results of maternal immune response to $N D V$.}

The maternal antibodies were evaluated by $\mathrm{HI}$ and ELISA assays.10 chicks obtained for one day old sampling (blood collected from heart) 5 of them used for HI test the result was $(112,117.8,116,6$ 113.4 and 115.2) and the other 5 chicks used for evaluation of ELISA antibody titer which was $(122,128,124,120,125)$.At $7^{\text {th }}$ day, 10 blood samples were obtained from different groups and tested for HI levels the results was $(58,59.6,57.2,55.8,54.4)$ and the ELISA antibody titer which was $(72,83,75,79,74)$ at the 14 th day maternal antibody mainly monitored throw the negative control group which left un-vaccinated and the result of $\mathrm{HI}$ titer decreased to $(14,20,18,16$ and 15), With parallel decrease on indirect ELISA antibody titer mean $(39,42,48$, 46 and 35 ). And at the $21^{\text {st }}$ day no titer could be detected as shown in table (2).

Results of vaccinations of chicken with different vaccine after priming with Hitchner B-1.

Vaccination of chicken in group II with LaSota vaccine at day 14 after priming with (Hitchner-B1) at day 7, resulted in increasing $\mathrm{HI}$ antibody titer mean from22.6 at day 14 to 35.6 at day $21,54.8$ at day 28 and 77.2 at day 35 with parallel increase ELISA antibody mean $\overline{\bar{K} \text { Kafrelsheikh Vet. Med. J. Vol. } 15 \text { No. } 1 \text { (2017) }}$ 
from130at day 14 to 160 at day 21,210 at day 28 and 280 at day 35 (Table -3). Vaccination of chicken in group III with Clone-30 vaccine at day 14 after priming with Hitchner-B1 at day7 showed similar behavior as the $\mathrm{HI}$ antibody titer mean was increased from18.8 at day 14 to 27.4 at day $21,49.8$ at day 28 and 58.6 at day 35. ELISA antibody titer mean was also increased from 122 at day14 to 145 at day 21, 194 at day 28 and 250 at day 35 (Table 4). Vaccination of chicken in group IV with ND 6/10 vaccine at day 14 after priming with (Hitchner-B1) at day 7, showed high level of HI antibody titer mean from 20.7at day 14 to32.4 at day 21,49.8 at day 28 and 62.7 at day 35 with parallel increase to ELISA antibody titer mean from 120 at day 14 to 152 at day 21,208 at day 28 and 260 at day 35. (Table-5).

\section{Results of challenge the chicken with virulent genotype VII NDV.}

Experimental challenge of 160 birds from various groups with virulent NDV resulted in death of 45 of 160 challenged birds. Among them, 10 out of 10 birds of control negative none vaccinated birds in group I, death of 12 out of 50 birds in group-II (Vaccinated with LaSota vaccine at day 14 after priming with Hitchner-B1 at day 7), death of 14 out of 50 challenged birds in group-III (Vaccinated with clone30 vaccine at day14 after priming with Hitchner-B1 at day 7), death of 9 out of 50 challenged birds in group-IV (Vaccinated with ND 6/10 vaccine at day14 after priming with Hitchner-B1 at day 7) (Table 7) no deaths on non- 
challenged control positive group confirm that deaths happened due to the effect of challenge virus.

\section{DISCUSSION}

The present study was aimed to investigate if genetic evolution of NDV is accompanied by antigenic evolution which may reduce the efficacy of current ND vaccines. To test this hypothesis, experimental birds were divided into 5 groups; each group was vaccinated with different vaccination protocol. The immune response to these different vaccines was monitored with $\mathrm{HI}$ and ELISA to evaluate vaccination efficacy. All groups were challenged with genotype VII NDV field isolate. Maternal antibodies to NDV detected in all groups by $\mathrm{HI}$ and ELISA test were higher in the 1st day of age and declined gradually until diminished at day 21 where, no titer could be detected. This result agrees with (GaffarElaminet al.,1990), who reported that maternal antibody detected by $\mathrm{HI}$ test at one day old chicks were higher than 2 weeks old broilers, and at 3 weeks of age, the HI antibody titer was very low or non-detectable. Primary vaccination of chicken in groups II, III, IV with HB1 vaccine at $7^{\text {th }}$ day via eye dropping leads to an increase in antibody titer mean from $(58,59.6,57.2,55.8)$ at day 7 to $(22.6,140.8,166.4)$ at day 14 of age, Also ELISA antibody titer increased from $(72,83,75,79)$ at day 7 to $(140,122,164)$ at day 14 . Unlike The mean antibody titers for negative control group of HI which decreased from 58 at day 77 to 16.6 at day 14 to undetectable value at day 21.and ELISA antibody titers which decreased from (72) at day 7 to 42 at day 14 to undetectable value at day 21.of age. Vaccination of chicks with LaSota vaccine at day 14 after priming with (Hitchner-B1) at day 7, revealed high $\mathrm{HI}$ antibody and ELISA titer mean at day 21, 28 and 35 day .In this respect our result had 
been agreed with those reported by (Bastami al.,1986), who reported that birds vaccinated with $\mathrm{HB} 1$ at 1st week of age followed by LaSota vaccine showed the highest titer (7.73) at 6th week of life. The same results was obtained with vaccination by Clone-30 vaccine at day 14 after priming with Hitchner-B1 at day7, in agreement with Alexander (2004), who reported that lentogenic cloned vaccines as clone 30 produced less vaccine reaction than LaSota- like virus with superior immunogenicity as compared to HB1-like virus.Also Vaccination with ND 6/10 vaccine at day 14 after priming with (Hitchner-B1) at day 7, showed an increased HI and ELISA antibody titers. This result agreed with (Cziffraet al.,1998), who reported that the Serological response after Immunization with an Asymptomatic ND virus strain patented as NDV- 6/10 Sera from 259 birds were examined by HI test and with BELISA before challenge (58\%) had at least an HI titer $\geq \log 21$, but only $(26 \%)$ had HI titers $\geq \operatorname{Iog} 23$, while $(64 \%)$ proved to be positive by ELISA.

The Experimental challenge of 160 birds from various groups with virulent NDV resulted in death of 45 of 160 challenged birds and this proved that vaccination with commonvaccinal strains might decrease shedding of vNDV shed as all belong to a single serotype but antigenic variation between genotype VII NDV and the commercial vaccinal strains prevent cross protection. Our results disagree with some studies as those reported by (Kapczynskiet al.,2013), who mentioned that under optimal experimental conditions, ensuring the administration of appropriate doses of vaccine and the sufficient time to induce an immune response, no statistical differences in morbidity and mortality rates between homologous and heterologous NDV vaccines have been observed. On the other hand, other studies agreed that although poor

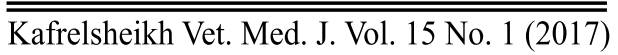


vaccine application or quality of the vaccines used cannot be excluded, there are indications that some ND outbreaks occurred despite intensive vaccination against NDV (Bogoyavlenskiy et al., 2009; Hassan et al., 2010). In addition, (Miller et al. 2009)suggested that, these outbreaks might be due to antigenic divergence between the vaccine strain and the circulating field strains.Many recent studies such as those carried out by (Huang et al. 2015) reported that although all NDVs belong to a single serotype, antigenic and genetic diversity between NDV isolates have been detected. (Diel et al. 2012) Found that, at least nine class I genotypes and 16 distinct lineages of class II NDV strains have been identified. Among these, viruses from genotype VII of class II are the predominant strains which have been associated with many of the most recent outbreaks world-wide. Also, many studies and ours have revealed that genotype VII NDVs have obvious differences to genotype II commercial vaccines or other genotypic strains in their genetics, immune response and protective efficacy (Miller et al., 2013).

Table (1): Schedule of experimental chicks vaccination programs

\begin{tabular}{|c|c|c|c|c|c|c|c|}
\hline \multirow{4}{*}{ Chicken age } & \multicolumn{7}{|c|}{ Chicken groups vaccination $\&$ sampling } \\
\hline & \multicolumn{4}{|c|}{1} & \multirow[b]{2}{*}{2} & \multirow[b]{2}{*}{3} & \multirow[b]{2}{*}{4} \\
\hline & \multicolumn{3}{|c|}{ Control positive } & $\begin{array}{c}\text { Control } \\
\text { negative }\end{array}$ & & & \\
\hline & 1st & $2^{\text {nd }}$ & 3rd & \multirow{2}{*}{ Sampling } & & & \\
\hline 7 days & HB1 & HB1 & HB1 & & HB1 & HB1 & HB1 \\
\hline 14 days & LaSota & Clone30 & ND 6/10 & Sampling & LaSota & Clone30 & ND $6 / 10$ \\
\hline $21 \& 28$ days & & & & \multicolumn{4}{|c|}{ Sampling only } \\
\hline 35 day & & & & \multicolumn{4}{|c|}{ Experimental challenge } \\
\hline
\end{tabular}

Table (2): The mean of ND maternalantibodytiters in chickens

\begin{tabular}{|c||c||c|}
\hline Chicken age & Mean ND-HI & Mean ND-ELISA \\
\hline \hline
\end{tabular}


protective efficacy of commercial new castle disease ...

Hasnaa Ahmed et., al.

\begin{tabular}{|c||c|c|}
\hline \hline & antibodytiter & Antibody titer \\
\hline \hline One day & 115 & 123.8 \\
\hline 7 days & 57 & 76.6 \\
\hline 14 days & 16.6 & 42 \\
\hline 21 days & 0 & 0 \\
\hline
\end{tabular}

Table (3): ND-HI and ELISA antibody titers in chickens Vaccinated with HB1 \& LaSota

\begin{tabular}{||c||c||c||c||}
\hline Chicken age & $\begin{array}{c}\text { Received } \\
\text { Vaccine }\end{array}$ & $\begin{array}{c}\text { Mean ND-HI } \\
\text { Antibody titer }\end{array}$ & $\begin{array}{c}\text { Mean ND -ELISA } \\
\text { Antibody titer }\end{array}$ \\
\hline \hline 7 days & HB1 & 59.6(Residual Of maternal immunity) & 83 \\
\hline 14 days & LaSota & 22.6 & 130 \\
\hline 21 days & - & 35.6 & 210 \\
\hline 28 days & - & 54.8 & 280 \\
\hline 35 days & - & 77.2 & 160 \\
\hline
\end{tabular}

Table (4): ND-HI and ELISA antibody titers in chickens Vaccinated with HB1\& colon-30

\begin{tabular}{|c|c|c|c|}
\hline Chicken age & Received vaccine & $\begin{array}{l}\text { Mean ND-HI } \\
\text { Antibodytiter }\end{array}$ & $\begin{array}{c}\text { Mean ND-ELISA } \\
\text { Antibody titer }\end{array}$ \\
\hline 7 days & HB1 & 57.2 (Residual of maternal immunity) & 75 \\
\hline 14 days & Colon-30 & 18.8 & 122 \\
\hline 21 days & - & 27.4 & 145 \\
\hline 28 days & - & 49.8 & 194 \\
\hline 35 days & - & 58.6 & 250 \\
\hline
\end{tabular}

Table (5): ND-HI and ELISA antibody titers in chickens Vaccinated with HB1\&ND6/10

\begin{tabular}{|c|c|c|c|}
\hline Chicken age & Received vaccine & $\begin{array}{c}\text { Mean ND-HI } \\
\text { Antibody titer }(\log 2)\end{array}$ & $\begin{array}{c}\text { Mean ND--ELISA } \\
\text { Antibody titer }(\log 2)\end{array}$ \\
\hline 7 days & HB1 & 55.8 (Residual of maternal immunity & 79 \\
\hline 14 days & ND $6 / 10$ & 20.7 & 120 \\
\hline 21 days & - & 32.4 & 152 \\
\hline 28 days & - & 49.8 & 208 \\
\hline 35 days & - & 62.7 & 260 \\
\hline
\end{tabular}

Table (7): Results of experimental challenge of different chicken groups

\begin{tabular}{|l||l|l||l||l|}
\hline Chicken groups & Number of challenged & Number of dead & Number of Survived & Protection \% \\
\hline
\end{tabular}

Kafrelsheikh Vet. Med. J. Vol. 15 No. 1 (2017) 


\begin{tabular}{|c|c|c|c|c|}
\hline & chickens & Chickens & chickens & \\
\hline I & 10 & 10 & 0 & 0 \\
\hline II & 50 & 12 & 38 & 76 \\
\hline III & 50 & 14 & 36 & 72 \\
\hline IV & 50 & 9 & 41 & 82 \\
\hline
\end{tabular}

\section{CONCLUSION}

Depending on the presented data, it is important to have a more detailed analysis of the biological and antigenic characters of currently circulating NDV strains and the efficacy of commonly used NDV vaccines for protection against the NDV isolates for better controlling the NDV in Egypt.

\section{REFERENCES}

- Alexander, D.J. 1998. Newcastle disease virus and other avian paramyxoviruses. In: Swayne DE, editor. A laboratory manual for the isolation and identification of avian pathogens. Kennett Square, PA: The American Association of Avian Pathologists, p. 156-63.

- Alexander, D.J. 2004. OIE Manual of Diagnostic Tests and Vaccines for Terrestrial Animals. Office of International Des Epizooties, Paris, p. 161.

- Alexander, D.J,Senne, D.A. 2008. Newcastle disease, other avian Paramyxoviruses, and pneumovirus infections, p 75-98. In Saif YM, Fadly AM, Glisson JR, McDougald LR, Nolan LK, Swayne DE(ed), Diseases of poultry, 12th ed. Blackwell Publishing, Ames, IA.

- Alexander, D.J., Aldous, E.W., Fuller, C.M. 2012. The long view: a selective review of 40 years of Newcastle disease research. Avian Pathol. 41: 329-335. 
- Al-Habeeb, M.A., Mohamed, M.H., Sharawi, S. 2013. Detection and characterization of Newcastle Disease Virus in clinical samples using real time RT-PCR and melting curve analysis based on matrix and fusion genes amplification, Vet. World. 6(5):239-243.

- Bastami, M.A,Amer, M.M., Hamouda,A.S.1986. Comparative study on the immune response of chickens vaccinated intramuscularly with different Newcastle disease vaccine. AssiutVet. Med. J. 17: 34-39.

- Bogoyavlenskiy, A., Berezin, V., Prilipov, A., Usachev, E., Lyapina, O., Korotetskiy, I., etal.2009. Newcastle disease outbreaks in Kazakhstan and Kyrgyzstan during 1998, 2000, 2001, 2003, 2004, and 2005 were caused by viruses of the genotypes VIIb and VIId. Virus Genes. 39(1):94-101.

- Cziffra, G., Meszaros, J., Horvath, E., Moving, V.,Engstrom,B. E. 1998. Detection of NDVspecific antibodies and the level of protection provided by a single vaccination in young chickens, Avian Pathology. 27(6): 562-565.

- Diel, D.G., da Silva, L.H., Liu, H., Wang, Z., Miller, P.J., Afonso, C.L. 2012. Genetic diversity of avian paramyxovirus type 1: proposal for a unified nomenclature and classification system of Newcastle disease virus genotypes. Infect. Genet.Evol. 12: 1770-1779.

- Dortmans, J. 2011. Virulence Determinants of Newcastle Disease Viruses Ph. D. thesis, Utrecht University, Nederland

- Elham, A., Pourbakhsh,.SA.,Ahmadi, M., Talebi, A. 2014.Pathotypic characterization of the Newcastle disease virus isolated from 
commercial poultry in northwest Iran. Turk. J. Vet. Anim. Sci. 38:383-387.

- GaffarElamin, M.A.,Khalafalla, A.I., Ahmed, S.M. 1990. Observation on the use of Komarov strain of Newcastle disease vaccine in the sudan. Trop. Anim. Hlth .prod. 25:151-154.

- Goldhaft, T.M., 1980. Historical note on the origin of the LaSota strain if Newcastle disease virus. Avian Dis. 24: 297-301.

- Hassan, W., Khair, S.A., Mochotlhoane, B., Abolnik, C. 2010.Newcastle disease outbreaks in the Sudan from 2003 to 2006 were caused by viruses of genotype 5d. Virus Genes. 40: 106-110.

- Hu, Z., Hu, S.,Meng, C., Wang, X., Zhu, J., et al. 2011. Generation of a genotype VII Newcastle disease virus vaccine candidate with high yield in embryonated chicken eggs. Avian diseases. 55: 391-397.

- Huang, Y., Yang, S., Hu, B., Xu, C., Gao, D., Zhu, M., et al. 2015. Genetic, pathogenic and antigenic diversity of Newcastle disease. Vet. Microbiol. 18:237-244.

- Kapczynski, D.R., Afonso, C.L., Miller, P.J. 2013. Immune responses of poultry to Newcastle disease virus. Dev.Comp.Immunol. 41: 447-53.

- Kim, L.M., King, D.J., Curry, P.E., Suarez, D.L., Swayne, D., Stallknecht, D.E., Slemons, R.D., Pedersen, J.C., Senne, D., Winker, K., Afonso, C.L. 2007a. Phylogenetic diversity among low-virulence Newcastle disease viruses from waterfowl and shorebirds and 
comparison of genotype distributions to those of poultry-origin isolates. J. Virol. 81:12641-12653

- Kim, L.M., King, D.J., Suarez, D.L., Wong, C.W., Afonso, C.L., 2007b. Characterization of class I Newcastle disease virus isolates from Hong Kong live bird markets and detection using real-time reverse transcription-PCR. J. Clin. Microbiol. 45: 1310-1314.

- Khan, S.T., Rehmani, R.C., Miller, P., Afonso, C.L. 2010. Phylogenetic and pathological characterization of Newcastle disease virus isolates from Pakistan. J. Clin. Microbiol. 48:1892-1894.

- Miller, P.J., King, D.J., Afonso, C.L., Suarez, D.L. 2007. Antigenic differences among Newcastle disease virus strains of different genotypes used in vaccine formulation affect viral shedding after a virulent challenge. Vaccine. 25: 7238-7246.

- Miller, P.J., Kim, L.M., Ip, H.S., Afonso, C.L. 2009. Evolutionary dynamics of Newcastle disease virus. Virology. 391: 64-72.

- Miller, P.J., Decanini, E.L., Afonso, C.L. 2010. Newcastle disease: evolution of genotypes and the related diagnostic challenges. Infect. Genet. Evol. 10: 26-35.

- Miller, P.J., Afonso, C.L., Attrache, J.E., Dorsey, K.M., Countney,S.C., Guo, Z., et al. 2013. Effects of Newcastle disease virus vaccine antibodies on the shedding and transmission of challenge viruses.Dev.Comp.Immunol. 41(4):505-13. 
- Voller, A.; Bidwell, D. E. and AnnBartlett. 1976. Enzyme immunoassay in diagnostic medicine, theory and practice. Bull. World Health Organ. 53: 55-65. 